\title{
Análisis del modelo didáctico de educación ambiental "La idea vector y sus esferas" desde el enfoque de los Objetivos de Desarrollo Sostenible. Un caso: la Escuela del Consumo de Cataluña
}

\author{
Genina Calafell \\ Departament de Didàctica de la Matemàtica i les Ciències Experimentals. Grup de \\ Recerca Cómplex. Universitat Autònoma de Barcelona. Rizoma Educació Transferència \\ de Coneixement. Spin off de la UAB, Barcelona. España.genina.calafell@uab.cat \\ ORCID: https://orcid.org/0000-0002-4026-7207

\section{Neus Banqué} \\ Departament de Didàctica de la Matemàtica i les Ciències Experimentals. Grup de \\ Recerca Cómplex. Universitat Autònoma de Barcelona. Rizoma Educació Transferència \\ de Coneixement. Spin off de la UAB, Barcelona.España.neus.banque@uab.cat \\ ORCID: https://orcid.org/0000-0003-3603-8636

\section{Queralt Grau} \\ Rizoma Educació Transferència de Coneixement. Spin off de la UAB, Barcelona. España. \\ qgrau@rizomaedu.com \\ ORCID: https://orcid.org/0000-0001-5025-7267
}

[Recibido: 17 Diciembre 2018. Revisado: 24 Enero 2019. Aceptado: 25 Enero 2019]

Resumen: La investigación analiza la introducción de la sostenibilidad en un programa educativo de Educación del Consumo mediante la incorporación de los Objetivos de Desarrollo Sostenible (ODS) de las Naciones Unidas. Para ello se valora la presencia o ausencia de los ODS y las competencias transversales para la sostenibilidad en la propuesta didáctica de la Escuela de Consumo de Cataluña, cuyo modelo formativo se orienta en el trabajo de los contenidos curriculares y las temáticas de la educación ambiental. Los resultados permiten diagnosticar si el modelo formativo favorece los objetivos de aprendizaje y competencias para avanzar en los ODS.

Palabras clave: Objetivos de Desarrollo Sostenible, Competencias Transversales, Educación del Consumo, Modelo Formativo

Valuation of an environmental education teaching model for consumption education through the perspective of the Sustainable Development Goals

Abstract: The research analyses the income of sustainability in an educational program about Consumption Education through the incorporation of Sustainable Development Goals (SDGs) from United Nations. For this purpose, it is valued the presence or absence of SDGs and the competences for sustainability in the teaching approach at the Consumption School of Catalonia, which educational model is guided by the work in curricular contents and topics from environmental education. The results allows for diagnosing the educational model as an assist of the learning goals and competencies to make progress with the SDGs.

Keywords: Sustainable Development Goals, Cross Competencies . Consumption Education, Teaching Model 
Para citar este artículo: Calafell, G., Banqué, N. y Grau, Q. (2019) Análisis del modelo didáctico de educación ambiental "La idea vector y sus esferas" desde el enfoque de los Objetivos de Desarrollo Sostenible. Un caso: la Escuela del Consumo de Cataluña Revista de Educación Ambiental y Sostenibilidad 1(1), 1302. doi: 10.25267/Rev_educ_ambient_sostenibilidad.2019.v1.i1.1302

\section{Introducción}

En los casi cincuenta años de trayectoria en Educación Ambiental las voces de fracaso o decepción son diversas por no conseguir disminuir o ablandar la crisis ambiental con mayor éxito (Gutiérrez, 2018). El mismo autor desde una mirada crítica y optimista diagnostica e identifica elementos propios del campo de la Educación Ambiental y también del contexto en el cual se desarrolla la Educación Ambiental como factores a considerar ante esta situación. Entre ellos, la falta de un modelo didáctico de Educación Ambiental.

Los modelos formativos en Educación Ambiental deberían proponer elaboraciones teóricas para que los profesionales de ésta los lleven a la práctica. Unas propuestas que deberían ir más allá de una actividad educativa centrada en la adquisición de hábitos y conductas para la sostenibilidad del medio e indagar en nuevas formas de aproximarse a los problemas ambientales desde un enfoque sistémico, global y con una visión crítica de ellos (Rodríguez y García, 2011). Una de las debilidades de la trayectoria de la EA y una oportunidad para dibujar nuevos alcances es la necesidad de profundizar más en la educación y su socialización y menos en como comprender el medio ambiente como objeto de estudio. Una idea que apunta Tilbury (2011) al advertir que la Educación Ambiental se ha focalizado en el qué y el para quién y ahora necesitamos poner el énfasis en el como desarrollar programas y proyectos eficaces de EA.

En esta misma línea, la investigación en educación ambiental nos muestra cómo existe una distancia entre las propuestas teóricas y sus aplicaciones en los contextos educativos que se refleja, por ejemplo, en la presencia de la educación ambiental en el currículo. Una incorporación de la educación ambiental en la enseñanza y aprendizaje que no ha encontrado formas óptimas de conectar las contribuciones ideológicas y las aportaciones prácticas generando propuestas didácticas dogmáticas o bien inhibidoras (Bonil, Calafell, Granados, Junyent, y Tarín, 2012). La inhibición de prácticas pro ambientales se da en instituciones que se caracterizan por una sólida elaboración documental y un discurso que asume la necesidad de introducir las grandes finalidades de la educación ambiental en el currículum educativo. Al mismo tiempo la presencia de la educación ambiental en la práctica del aula es nula, anecdótica o estereotipada. El dogmatismo surge de una relación absolutamente rígida entre el discurso ideológico y el práctico. Son instituciones que han elaborado un sólido marco ideológico que acaba cerrándose en si mismo tomando el carácter de discurso único. De este modo, una vez elaborado, el discurso ideológico pasa a tener carácter de dogma que se debe transferir en la actividad docente: la transmisión de la información en forma de consignas cerradas para logar un cambio de actitudes. Entre los dos extremos se pueden caracterizar diversidad de modelos formativos que manifiestan multitud de variaciones en la relación entre el discurso ideológico y el práctico. 
El Grupo de Investigación Còmplex asume la necesidad de investigar e innovar en el cómo de la educación ambiental a partir de la propuesta de un modelo didáctico para favorecer la introducción de la educación ambiental en los programas educativos. Un modelo didáctico que toma como referente los principios de la complejidad, la interdisciplinariedad y los enfoques sociocríticos. Para este cometido la investigación se ha favorecido de un contexto educativo como es la Escuela del Consumo de Catalunya de la Agencia Catalana del Consumo (Generalitat de Catalunya). Un espacio para la educación del consumo que ha sido clave para favorecer un diálogo entre la investigación, la innovación y la implementación de actividades educativas. Un crisol que en palabras de Sauvé (2015) se convierten en contextos necesarios para aprovechar espacios de la vida cotidiana de las comunidades para desarrollar acciones de educación ambiental y fortalecer la colaboración entre instituciones.

Después de quince años de investigación y práctica, los responsables del proyecto de la Escuela del Consumo de Catalunya convergen en la necesidad de evaluar el proyecto y definir nuevas metas y horizontes. En esta diagnosis, una de las perspectivas que se consideran relevantes, aunque no la única, es valorar la introducción de la sostenibilidad en el programa educativo de la ECC. Para ello se aprovecha el marco que proporciona la definición de los diecisiete Objetivos para el Desarrollo Sostenible (ODS) de las Naciones Unidas.

En consonancia con esta necesidad, nos proponemos explorar (1) qué modelo educativo de EA para la educación del consumo, y (2) qué planteamientos y líneas educativas sugieren los Objetivos para el Desarrollo Sostenible.

\section{Un modelo educativo de Educación Ambiental para la educación del Consumo.}

Los modelos didácticos aparecen necesariamente como modelos abiertos y dinámicos. Abiertos ya que se muestran influenciados por las corrientes de pensamiento, por los resultados de la investigación y por los datos empíricos de la propia experiencia. Reflejan sus limitaciones y posibilidades en un continuo diálogo con su entorno huyendo de planteamientos reduccionistas, pero reivindicando de forma permanente su capacidad de entender e intervenir sobre una fracción del mundo. Son dinámicos porque tienen la capacidad de cambiar con las influencias del entorno. Son capaces de moverse en el conflicto continuo que presenta el diálogo entre estabilidad y cambio. Estabilidad ya que presentan unos principios identitarios, unos fundamentos que favorecen que se muestren coherentes, válidos y fiables. Pero a la vez son capaces de adecuarse a un mundo que plantea de forma continua nuevos retos. En este sentido son capaces de adecuarse a nuevas necesidades, nuevos fenómenos a tratar y a nuevos perfiles de contextos educativos. Desde esta premisa definimos un modelo didáctico que pueda ofrecer herramientas para un cambio significativo en la forma de trabajar los contenidos curriculares y las temáticas propias de la educación ambiental. Un modelo que toma como eje vertebrador los principios de la complejidad (Bonil et al., 2004) y que se construye en los principios que han orientado históricamente la educación ambiental. Esta propuesta toma el reto de huir del riesgo de moralizar la educación ambiental y dar empoderamiento y oportunidades individuales y colectivas para la transformación hacia nuevas formas de pensar y actuar en el mundo. 
La propuesta formativa que se presenta, denominada La idea vector y sus esferas (Bonil, Calafell, Granados, Junyent, y Tarín, 2012; Calafell, Junyent y Bonil, 2015; Calafell y Junyent, 2017) se fundamenta en tres principios esenciales: (1) la transformación del entorno desde la incorporación de la relación medio y sociedad para la sostenibilidad; (2)el desarrollo del pensamiento crítico y creativo de los alumnos desde un diálogo entre ser profesional y ser ciudadano; (3) el empoderamiento de los alumnos, los profesores y las instituciones (Figura 1).

Esta propuesta se fundamenta en una Idea Vector, que es motor de la actividad y las esferas conceptual, metodológica, creativa y de investigación que la concretan en el escenario del aula. Todo ello considerando que la relación tiempo - espacio - contexto enmarcan la acción de educación ambiental y se concibe como una propuesta abierta a cambios: dinámica y en evolución.

Para comprender una acción en educación ambiental pensamos que es necesario considerar dónde se desarrolla, cuándo se desarrolla y qué significado tiene para sus participantes. De allí la importancia de considerar los ejes espacio, tiempo y contexto y su interacción para construir y compartir entre educadores y participantes de la actividad una forma de entender, ser y actuar en el mundo.

Una cosmovisión que, como una bolsa de té, infusa toda la acción educativa y que se trabaja a partir de una idea vector. Esta idea principal orienta y vertebra toda la acción educativa y es una manera de aproximarse al mundo desde la transformación. Metafóricamente, podrían ser las gafas que ponemos a los participantes de la acción educativa para que sean capaces de ver en su entorno nuevas entidades $\mathrm{y}$ relaciones. La idea vector es transversal a las áreas de conocimiento, incorpora la

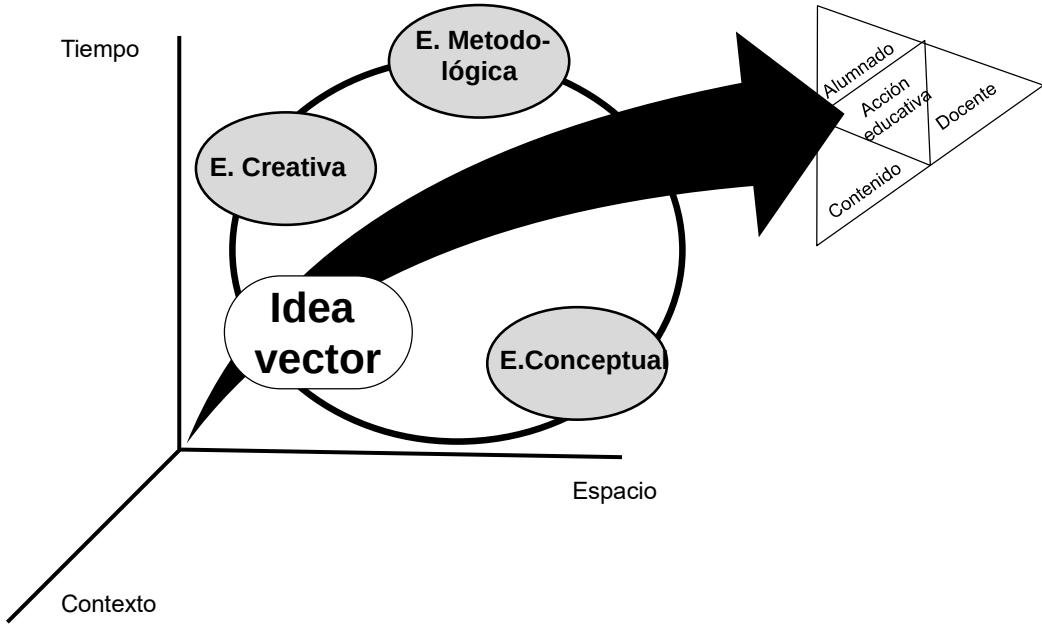

Figura 1. Modelo didáctico de la idea vector y sus esferas perspectiva de la complejidad y tiene un fuerte componente social. Para pensar y seleccionar una idea vector hay que ir más allá de conceptos curriculares y evitar valores éticos para no caer en contenidos o moralejas preestablecidas o dogmáticas.

La esfera conceptual, metodológica y creativa ayudan a concretar la idea vector en la acción educativa considerando los contendidos a enseñar y aprender, el rol del profesor y el protagonismo del alumnado. Estas esferas están íntimamente relacionadas entre ellas a modo de engranaje y se retroalimentan entre sí. La esfera conceptual conecta la idea vector con los contenidos curriculares de la acción formativa. Debe tener un claro y compartido significado para el colectivo al que se 
dirige la acción educativa y permitir estimular la reflexión y la acción en torno al mundo y la cotidianidad de los participantes. La esfera creativa aporta una visión cultural a la acción didáctica desde una perspectiva estética, artística y transformadora. Esta dimensión implica que se considera una perspectiva divergente (o aparentemente no tradicional) en la comprensión del mundo y de su relación con los contenidos que se trabajan. La esfera metodológica lleva a la reflexión sobre las opciones de cómo se realizará la acción formativa y qué estrategias didácticas se utilizarán en la actividad (la dinámica de aula, los instrumentos didácticos, la organización de los participantes, el rol del profesorado, la temporización y secuenciación de la acción o la forma de evaluar).

La Idea vector y las esferas se proyectan sobre la actividad educativa y ésta se convierte por lo tanto en una experiencia creada por sus actores, donde docentes, alumnado y currículum se encuentran en una construcción efímera modulada por cuatro esferas que conectan el conjunto con una gran finalidad en términos de cambio de cosmovisión. El aula se convierte en un espacio constructor y construido, un espacio de diálogo entre agentes de gran fuerza comunicativa en que sobre todo se espera que pasen cosas en el sentido más amplio de la expresión. Un espacio donde se opta por un currículum que dialoga entre la especificidad de las áreas de conocimiento, sus modelos explicativos, y los fenómenos del mundo sobre los que proyecta su conocimiento la ciudadanía. La actividad se convierte en un espacio de aprendizaje híbrido (Wals, 2013) en el que hay permeabilidad entre disciplinas, sinergia entre distintos actores y la abertura a nuevas formas de pensar, hacer y actuar.

\section{Unas estratégias metodológicas para diseñar actividades de educación el consu- mo desde el Modelo Idea vectora y sus esferas.}

Cuando se concreta esta propuesta en el diseño de actividades y la búsqueda de estrategias metodológicas encontramos pertinente orientar la actividad desde: la definicion de contextos y fenómenos didácticos, la formulación de preguntas, el diálogo disciplinario y la elección y selección de contendidos estructurantes. Estas herramientas se han ido implementado en la ECC desde una práctica reflexiva y de innovación, retroalimentando y enriqueciendo su aplicación en las actividades educativas a partir de la investigación del Grupo de Investigación Cómplex. Para poder conectar la propuesta de herramientas con las ejemplificaciones prácticas el lector puede consultar experiencias educativas que se han divulgado en artículos prácticos (Calafell, Banqué y Viciana, 2019; Gómez, Fonolleda y Calafell, 2013; Banqué, Querol y Calafell, 2011; Calafell y Bonil, 2010)

\section{Definición de un contexto y un fenómeno didáctico.}

Tradicionalmente el currículo se ha organizado tomando ejes temáticos concretos que se han considerado propios de las disciplinas. La selección de un fenómeno del mundo para empezar una programación de educación del consumo es una oportunidad para dejar de trabajar por temáticas concretas y estáticas y presentar a los niños y niñas fenómenos dinámicos y que se adaptan a los cambios del mundo. Estos fenómenos tienen un triple significado (Develay, 1995): para el currículum (en éste caso de ciencias), para los niños y niñas y para el contexto social. Cuando contextualizamos un fenómeno didáctico, tomamos una fracción del mundo relevante para el alumnado y lo llevamos al aula con toda su complejidad. Es aquella parte del 
mundo, de la cotidianidad del niño, que conecta con el aprendizaje de la escuela y con el entorno social y cultural que nos rodea.

Formulación de una pregunta mediadora

La capacidad de formularse preguntas aparece como una competencia fundamental de la educación ambiental y la educación del consumo pues ellas permiten imaginarse nuevos escenarios futuros, una competencia clave para la educación de futuros contextos más sostenibles y justos. Desde esta perspectiva se consideran oportunas las preguntas generadoras o mediadoras (Márquez et al., 2004) que constituyen puentes entre la forma de ver el mundo del alumnado y el modelo conceptual que se trabaja. Deja de tener sentido construir un saber enciclopédico y transmisor que busca respuestas cerradas y sumativas y se considera crucial la formulación de preguntas donde la idea de proceso es relevante.

Incorporación del Diálogo Disciplinario

Las disciplinas son un elemento esencial de la actividad educativa que conectan el conocimiento teórico con nuestro patrimonio cultural y permiten orientar la acción educativa desde el rigor. La diversidad de disciplinas para interpretar un fenómeno del mundo convierte las disciplinas en un instrumento potente para formar a la ciudadanía activa, crítica y responsable. El diseño de actividades desde un fenómeno del mundo o una fracción se convierte en un espacio de encuentro de conocimientos disciplinares que dialogan para construir un conocimiento complejo y transversal. El diálogo disciplinario (Calafell y Bonil, 2010) en el diseño de actividades permite estimular la diversidad de lenguajes y una visión creativa del mundo.

La definición de los contenidos estructurantes

La tradición de educación del consumo se ha fundamentado en la transmisión de información sobre los mecanismos organizadores de la sociedad del consumo. Información sobre el marco legal, los códigos de la publicidad. Esta visión tiene como obstáculo la dificultad de adaptarse a los cambios que se dan en la sociedad del consumo. Delante de esta dificultad trabajar sobre contenidos estructurantes puede ser una forma de orientar la acción didáctica. Los contenidos estructurantes son aquellos que permiten construir un patrón conceptual de consumo (Bonil, Calafell, Querol, Fonolleda y Pujol, 2013). Son contenidos que permiten al alumnado situarse delante de cualquier fenómeno y construir su forma de sentir, pensar y actuar en clave de complejidad. Tomar como referencia los contenidos estructurantes conlleva organizar el currículum de la educación del consumo para favorecer que el alumnado pueda construir patrones de consumo delante de los fenómenos que le son significativos. Comporta decidir qué contenidos se pueden introducir en cada momento y qué tipos de relaciones se pueden establecer desde las actividades orientadas a la educación del consumo.

\section{Los Objetivos para el Desarrollo Sostenible}

La definición de los ODS fija una nueva agenda para el 2030 e invita a las instituciones y a la gobernanza a asumir su responsabilidad para lograr los diecisiete objetivos y las 169 metas. En este marco las instituciones deben plantear estrategias para desarrollar los ODS con sus recursos y la Agencia Catalana del Consumo (ACC) como organismo competente en materia de consumo asume este cometido. La agenda para 
el 2030 contempla la educación como una estrategia clave y esencial para lograr este horizonte. La educación ambiental debe responder a la realidad urgente de la problemática ambiental desde la definición no únicamente de nuevos objetivos y contenidos pertinentes sino especialmente, de pedagogías que empoderen a la ciudadanía y a los estudiantes. Desde este prisma, la ACC establece que el proyecto educativo de la ECC se convierte en un contexto esencial para la incorporación de los ODS en su gobernanza.

Los ODS no están libres de controversias y voces de escepticismo por sus acuerdos utópicos y no vinculantes jurídicamente que favorece un horizonte hipotético con una carencia de responsabilidades precises y de incumplimiento de las 169 metas. A la vez, su definición enmascara la gravedad de la crisis sistémica que choca con los datos existentes sobre el incremento de la desigualdad, el autoritarismo y la violencia divulgando un diagnóstico optimista así como una metas que no cuestionan el sistema económico neoliberal (Garcia, 2018, CNEA). En este sentido, la Educación Ambiental se enfrenta a lo que se ha metaforizado como el manto de Penélope en el que Penélope presionada por su entorno, tejía un manto para Ulises que, se suponía había muerto. La esperanza de verlo vivo hacia que, durante el día y a ojos de la gente, tejiese el manto y por la noche lo deshiciese. Un paralelismo que explica que lo que la EA se afana en ir construyendo el sistema hegemónico lo destruye (Calvo y Gutiérrez, 2007, en Gutiérrez Bastida, 2018, pág. 89).

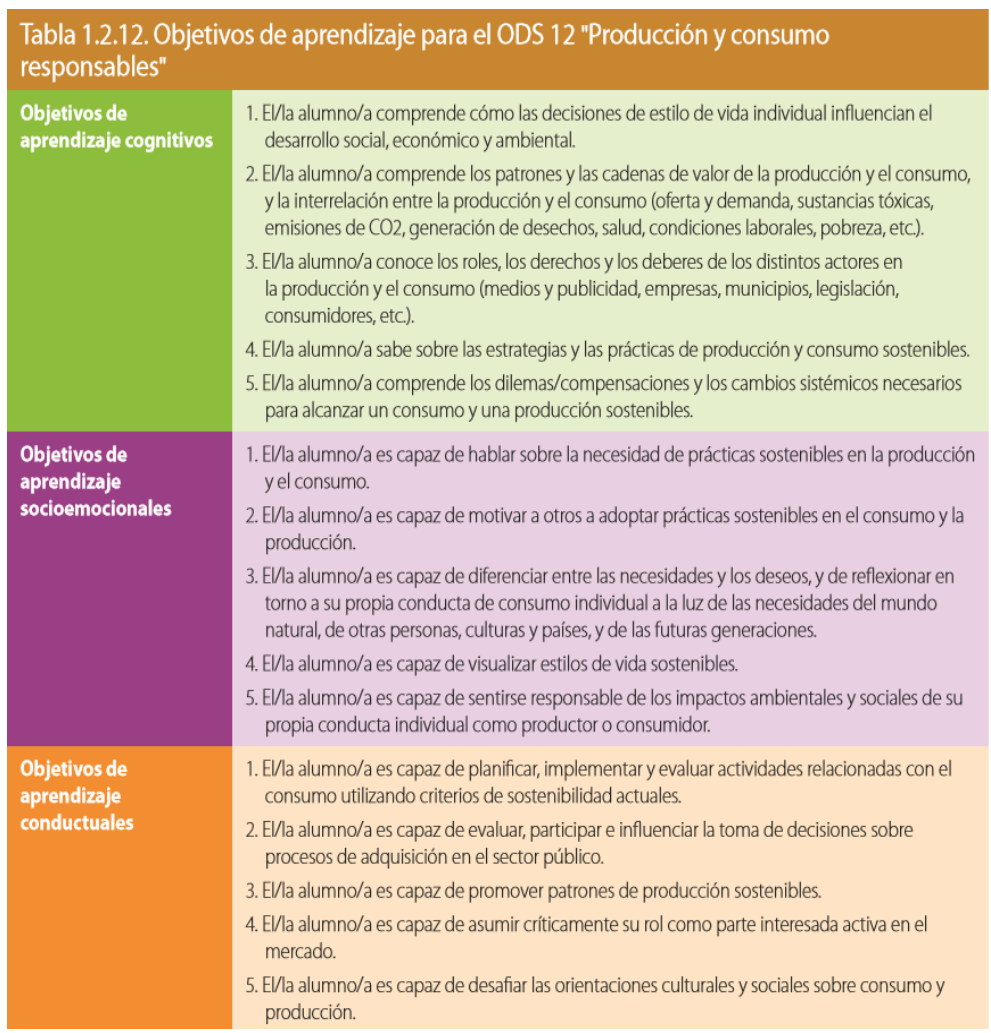

Figura 2. Ejemplo de los Objetivos de Aprendizaje para el ODS 12. Fuente: http://unescocat.org/wp-content/uploads/2018/02/Educació-per-al-DesenvolupamentSostenible_-Objectius-daprenentatge-CAT.pdf 
A la vez la aprobación de los ODS ha favorecido que muchas instituciones hayan impulsado interesantes estudios y mapeos en los que se exploran las capacidades y desafíos de cada una, analizando estrategias institucionales necesarias y proyección de ellas a partir de los resultados. (Gómez, 2017/2018). Es desde este marco que la Agencia Catalana del Consumo, reconociendo las limitaciones de los ODS explora también sus capacidades para desarrollar estrategias educativas necesarias para avanzar hacia sociedades más sostenibles. Desde esta visión la investigación pretende realizar un mapeo y una diagnosis de como se incorporan los ODS en el proyecto educativo de la Escuela del Consumo de Catalunya. En este proceso y al ser una institución que su objetivo final es educar en consumo se ha focalizado el estudio en la incorporación de los objetivos de aprendizaje que la UNESCO a definido para logar los ODS y el alcance de las competencias transversales clave para logar los ODS. (Ver figura 2 y 3 ).

Para ello la investigación pretende:

- Describir la incorporación de los objetivos de aprendizaje en las actividades educativas de la

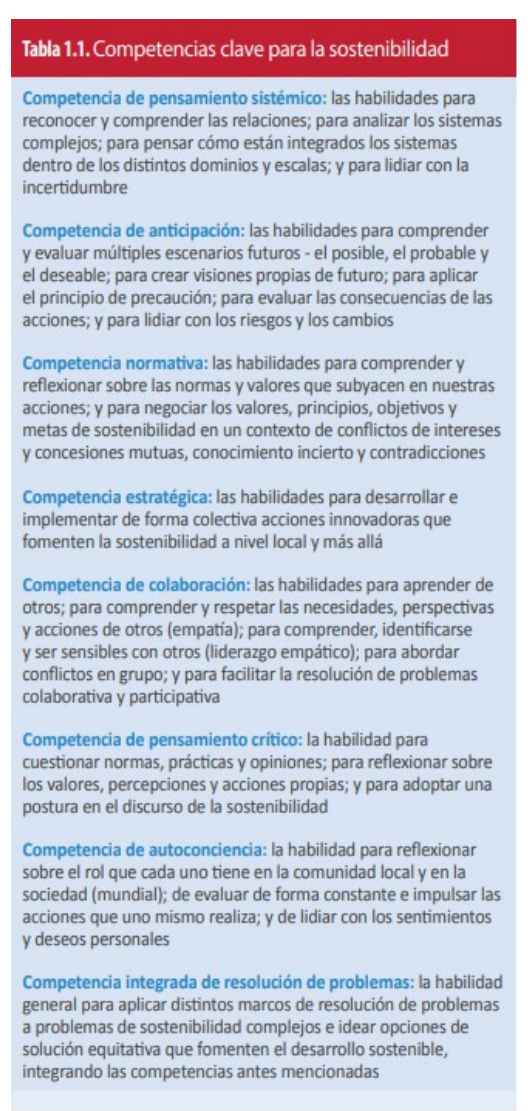

Figura 3. Competencias transversales de los Objetivos de Aprendizaje para el ODS 12. Fuente: http://unescocat.org/wpcontent/uploads/2018/02/Educació-per-alDesenvolupament-Sostenible_-Objectiusdaprenentatge-CAT.pdf Escuela del Consumo de Catalunya,

- Describir la incorporación de las competencias transversales en las actividades educativas de la Escuela del Consumo de Catalunya,

La contribución del artículo es diagnosticar y tomar decisiones entorno a si el modelo didáctico que se ha tomado como referente para el diseño de las actividades educativas favorece o inhibe objetivos de aprendizaje y competencias transversales para avanzar en las ODS.

\section{L'escola del Consum de Catalunya, un contexto para la investigación.}

Esta investigación se desarrolla en el marco de las actividades que lleva a cabo la Escuela del Consumo de Cataluña (ECC) de la Agencia Catalana del Consumo (ACC), como centro permanente de educación en consumo (www.consum.cat).

La ACC es un organismo autónomo de la Generalitat de Catalunya cuyo objetivo es garantizar los derechos de las personas como consumidoras de bienes y productos y usuarias de servicios. Entre los diferentes objetivos de la ACC, destaca el de formar, 
informar y educar a las personas consumidoras y es en este contexto que en 2003 se crea la ECC. La ECC ha ido consolidando su forma propia de entender la educación en consumo, una educación que conlleva a plantearse el reto de favorecer la formación de una ciudadanía crítica, activa y responsable:

Crítica, para que, ante los actos de consumo, sea capaz de efectuar un análisis, de formarse una opinión y de contrastar puntos de vista de manera constructiva.

Activa, porque, en una sociedad democrática, la formación de las personas debe estar orientada a actuar en relación con el medio. Desde esta perspectiva, un acto de consumo es una forma de actuar en la que existen diferentes momentos: desde decidir que se tiene una necesidad y escoger un producto hasta que llega el momento en que hay que utilizar y mantener el producto adquirido.

Responsable, porque permite construir el propio modelo de consumidor capaz de asumir sus decisiones, de ser consciente de las consecuencias de sus acciones sobre el medio y de medir su impacto. Un consumidor con visión global es capaz de entender que los actos de consumo tienen dimensiones que evolucionan en el tiempo y el espacio, y que, por tanto, deben realizarse de manera reflexiva.

La ECC actualmente es un referente en el campo de la educación del consumo por su espacio de confluencia de la investigación, la innovación y la docencia. La investigación en educación del consumo (aportaciones teórica y didácticas y estudio de los hábitos de consumo de los jóvenes) enriquece la indagación de nuevas metodologías y estrategias didácticas para introducir la educación del consumo en el currículum escolar. La investigación y la innovación aportan un valor de calidad a la actividad docente llevada a cabo diariamente en el ámbito geográfico catalán a través de talleres dirigidos al alumnado de educación primaria, de educación secundaria obligatoria, de bachillerato, de ciclos formativos (Figura 4).

\begin{tabular}{|c|c|c|c|}
\hline & Ref. & Taller & contenido \\
\hline \multirow{8}{*}{$\begin{array}{c}\text { Talleres para } \\
\text { la educación } \\
\text { Primaria }\end{array}$} & P1 & Veo, veo... qué tienda es? & La diversidad de tiendas en el barrio \\
\hline & $\mathrm{P} 2$ & Ñam, ñam...que hay para merendar? & Las meriendas \\
\hline & P3 & ¿Me hago mayor? & Las fiestas de cumpleaños \\
\hline & $\mathrm{P} 4$ & ¿A que jugamos? & Los juguetes \\
\hline & P5 & ¿Como se mueve la energía por la escuela? & La gestión de la energía en la escuela \\
\hline & P6 & ¿Qué hacemos esta tarde? & La gestión del tiempo libre \\
\hline & P7 & ¿Me compras una sonrisa? & Las emociones en la compra de productos \\
\hline & P8 & ¿Qué valor tiene la energía que consumimos? & La gestión de la energía en un día de un niño/a \\
\hline \multirow{14}{*}{$\begin{array}{l}\text { Tallers para } \\
\text { la educación } \\
\text { Secundaria }\end{array}$} & S1 & ¿Me sirven los servicios? & El uso y la contratación de servicios \\
\hline & S2 & ¿Dónde tengo mi dinero? & La gestión cotidiana que hacen los jóvenes de su dinero \\
\hline & S3 & ¿El agua está siempre en equilibrio? & El uso i la gestión cotidiana del agua \\
\hline & S4 & ¿Mensajes o mensajeros? & La gestión de la publicidad en la vida cotidiana de los jóvenes \\
\hline & S5 & ¿Cómo sé que compro? & La compra de productos alimentarios en función del etiquetado \\
\hline & S6 & ¿El chocolate es dulce para todos? & El consumo de chocolate \\
\hline & S7 & ¿Qué reglas tiene el consumo? & Los consumidores delante de los conflictos de consumo \\
\hline & S8 & ¿Vamos de compras? & $\begin{array}{l}\begin{array}{l}\text { La compra de ropa y tecnología como un reflejo del tipo de } \\
\text { consumidor }\end{array} \\
\end{array}$ \\
\hline & S9 & ¿Qué me pongo esta mañana? & Criterios en la compra de ropa \\
\hline & $\mathrm{S} 10$ & Es sábado, ¿qué hacemos? & La gestión del tiempo libre \\
\hline & S11 & ¿Me siento bien? & Los estilos de vida saludables \\
\hline & S12 & ¿Siempre vemos la energía que consumimos? & $\begin{array}{l}\text { La gestión de la energía en los aparatos tecnológicos (móvil i } \\
\text { portátil) }\end{array}$ \\
\hline & $\mathrm{S} 13$ & ¿Impactos o mensajes? & La gestión de la publicidad en la vida cotidiana de los jóvenes \\
\hline & S14 & Internet: ¿consumimos o nos consumen? & La cotidianidad del entorno virtual de los jóvenes \\
\hline
\end{tabular}

Figura 4. Actividades educativas ofrecidas por la Escuela del Consumo de Catalunya (ECC)http://consum.gencat.cat/ca/lagencia/escola-del-consum-de-catalunya/docencia/ 


\section{Metodología de la investigación}

La presente investigación se plantea desde el paradigma interpretativo, con el fin de describir y comprender aquello que es único del contexto de la Escuela del Consumo de Catalunya, más que lo generalizable, y en interpretar la realidad educativa des del significado de las personas implicadas en el contexto (Arnal, 2000).

Bajo este paraguas se enmarcan las decisiones metodológicas más importantes de la investigación: la definición del problema de investigación, la muestra o la recogida y análisis de datos, entre otras (Colás Bravo y Buendía Eisma, 1998).

La muestra elegida para la investigación es pequeña en cantidad, pero rica en diversidad y matices. La combinación de diferentes fuentes para la obtención de información permite la valoración de diversos enfoques sobre qué ámbitos de la sostenibilidad se trabajan o son susceptibles de trabajarse en un futuro en la ECC. A continuación, se presentan las tres fuentes de información utilizadas:

- Los responsables de la gestión educativa de la ECC, que coordina y dinamiza la institución educativa según las directrices de la Agencia Catalana del Consumo y tienen una visión estratégica sobre la línea de Educación del Consumo de la institución (2 participantes).

- El equipo educativo de la ECC, que realiza diariamente los talleres y las actividades docentes con el alumnado que visita la institución y tienen un conocimiento cotidiano y metodológico sobre las actividades de Educación del Consumo de la institución (6 participantes).

- Las Guías docentes de las actividades educativas de la ECC, que aportan una información objetiva clave para describir y comunicar la programación de la Educación del Consumo en cada actividad: duración del taller, cursos a los que va destinado, dinámica que se lleva a cabo, contenidos estructurantes y curriculares y orientaciones didácticas. Estas guías están a disposición de cualquier centro educativo, para facilitar la elección de las actividades adecuadas a sus necesidades (22 Guías, correspondientes a los 8 talleres de Educación Primaria y a los 14 de Educación Secundaria).

El proceso de recogida de datos para estas tres fuentes de información se ha basado en dos técnicas: la entrevista semiestructurada y el análisis documental. La entrevista semiestructurada se ha utilizado para obtener la información de los responsables de la gestión educativa de la ECC y del equipo educativo, y el análisis documental para obtener la información de las Guías docentes de las actividades de la ECC.

En el primer caso, la recogida de datos a partir de la entrevista se plantea como una sesión grupal de los responsables de la ECC (2p) y otra del equipo educativo (6p), con el objetivo de compartir y argumentar la diversidad de visiones sobre los contenidos de cada taller. La entrevista se centra en primer lugar, en cuáles son los ODS que se movilizan en los talleres; en segundo lugar, en la discusión de cada taller por separado para valorar cuantos ODS creen que se incorporan y de qué manera los ven representados dentro de las dinámicas que se realizan; y finalmente, en las competencias transversales que creen que se trabajan en los talleres. 


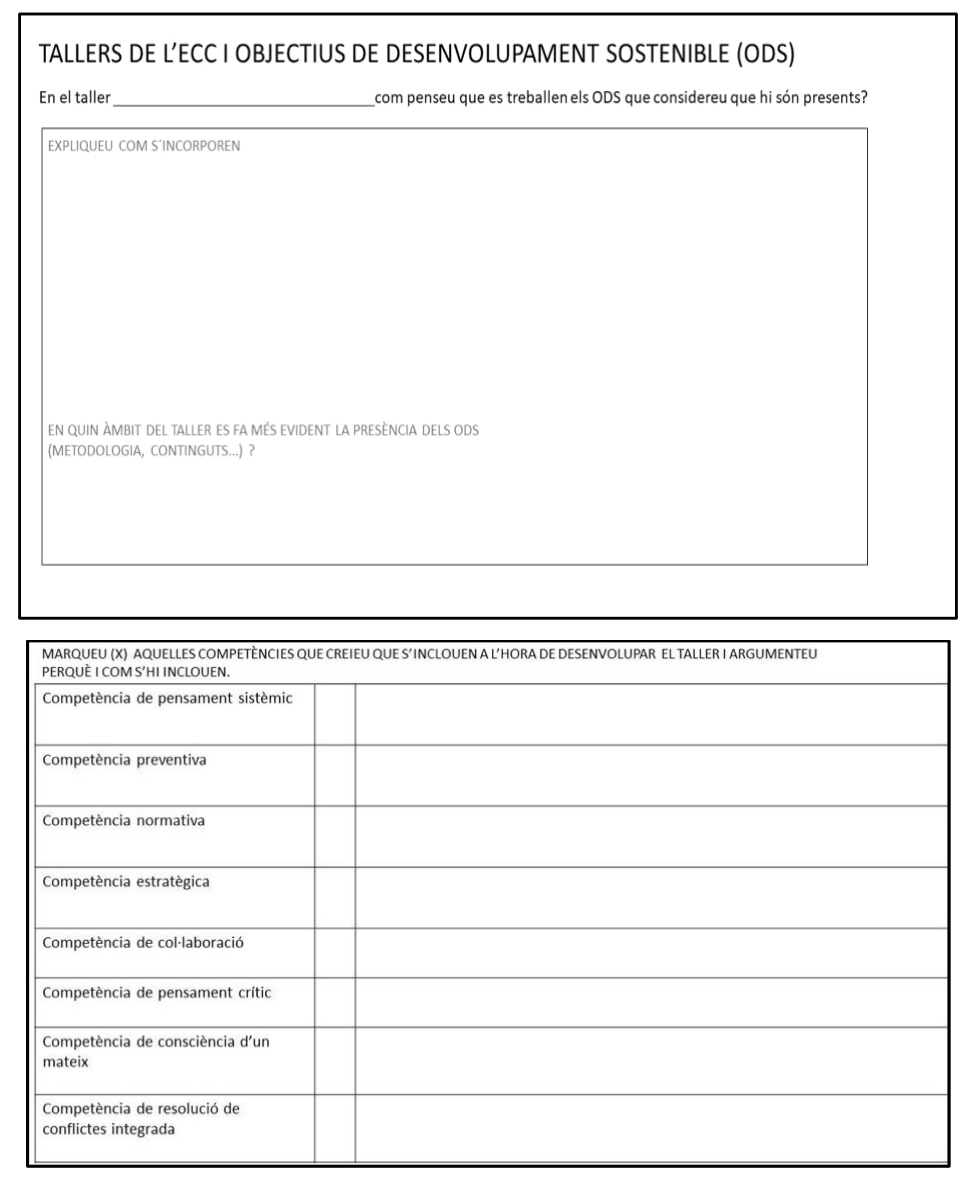

Figura 5. Documento de recogida de datos.

En esta sesión tanto los responsables como los educadores tenían que dar respuesta por escrito a las preguntas planteadas, así como anotar en un "diario" todas aquellas reflexiones que compartían para dar respuesta a las preguntas de la entrevista. Los datos utilizados para el análisis de la información son las respuestas recogidas de los participantes, así como sus diarios de entrevista. A continuación, se muestran los documentos de preguntas que respondían los entrevistados (Figura 5).

En el segundo caso, la recogida de datos a partir del análisis documental se plantea desde el análisis de contenido de las Guías docentes de las actividades de Educación Primaria (8 Guías) y de las actividades de Educación Secundaria (14 Guías). El objetivo es identificar en los apartados de Contenidos estructurantes y Orientaciones metodológicas la relación con los Objetivos de Aprendizaje por cada ODS, clasificados según el dominio cognitivo, socioemocional y conductual, como marca el documento oficial de la UNESCO. Los datos utilizados para el análisis de la información son segmentos de significatividad que recogían las Guías en relación al foco de la investigación. A continuación, se muestra un ejemplo de Guía docente de la actividad ¿Qué me pongo esta mañana? (Figura 6)

El análisis de una realidad compleja requiere la combinación de diferentes técnicas de investigación y métodos para conseguir resultados complementarios y a la vez más globales (Rodríguez y Gutíerrez, 2005). En este proceso se ha basado la investigación presente, en un proceso de triangulación de datos (Denzin, 1970) para llevar a cabo el 
análisis, con el fin de aumentar la validez de los resultados y reducir el sesgo del análisis parcial de los datos.
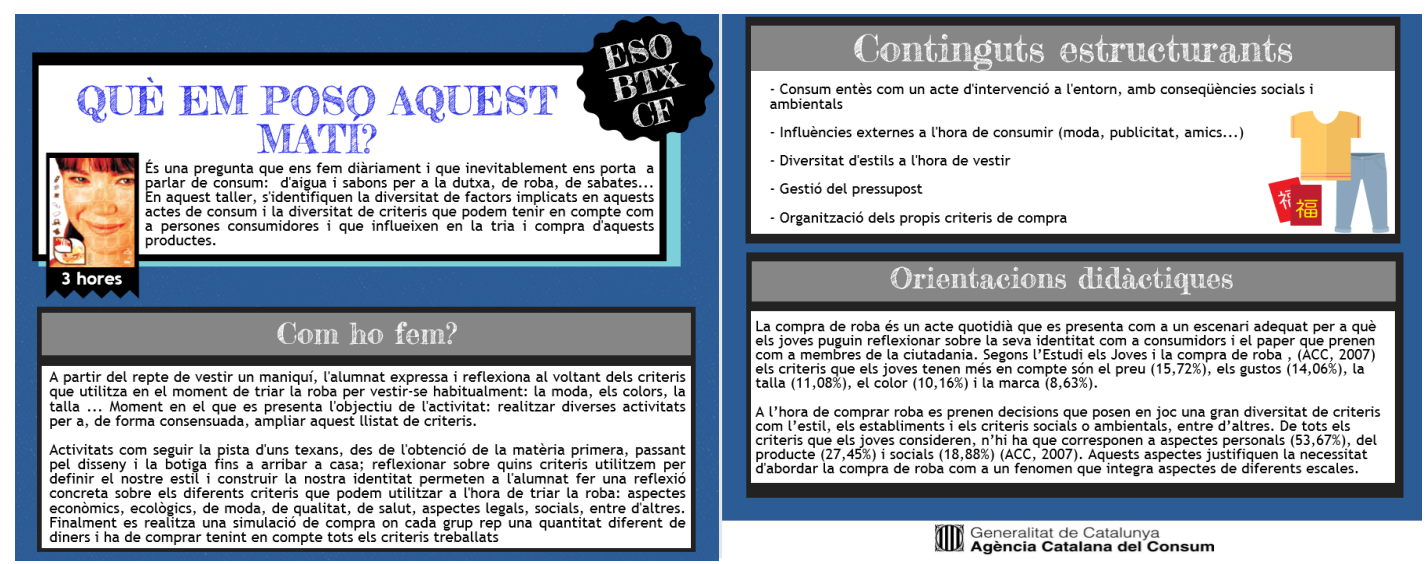

Figura 6. Ejemplo de una guía docente

\section{Análisis de datos}

A partir de los objetivos de aprendizaje de cada ODS se ha realizado una tabla para cada uno de los talleres para aceptar la presencia o la ausencia de cada ODS en los contenidos de las actividades educativas.

\begin{tabular}{|c|c|c|}
\hline \multicolumn{3}{|c|}{ ODS 12 Producción y consumo responsables } \\
\hline \multicolumn{3}{|r|}{ ¿Qué me pongo esta mañana? } \\
\hline $\mathrm{OA}$ & Presencia & Evidencias \\
\hline \multirow{2}{*}{ CG1 } & \multirow{2}{*}{$x$} & $\begin{array}{l}\text { Consumo entendido como un acto de intervención en el entorno, con consecuencias } \\
\text { sociales y ambientales. (CE) }\end{array}$ \\
\hline & & Abordar la compra como un fenómeno que integra aspectos de diferentes escalas (OD) \\
\hline CG2 & $x$ & $\begin{array}{l}\text { Consumo entendido como un acto de intervención en el entorno, con consecuencias } \\
\text { sociales y ambientales. (CE) }\end{array}$ \\
\hline \multicolumn{3}{|l|}{ CG 3} \\
\hline CG 4 & $x$ & $\begin{array}{l}\text { Consumo entendido como un acto de intervención en el entorno, con consecuencias } \\
\text { sociales y ambientales.(CE) }\end{array}$ \\
\hline CG5 & $x$ & $\begin{array}{l}\text { Consumo entendido como un acto de intervención en el entorno, con consecuencias } \\
\text { sociales y ambientales. Organización de los propios criterios de compra.(CE) }\end{array}$ \\
\hline \multicolumn{3}{|l|}{ SE 1} \\
\hline SE2 2 & $x$ & Influencias externas a la hora de consumir.(CE) \\
\hline SE 3 & $x$ & $\begin{array}{l}\text { Consumo entendido como un acto de intervención en el entorno, con consecuencias } \\
\text { sociales y ambientales. Organización de los propios criterios de compra.(CE) }\end{array}$ \\
\hline SE 4 & $x$ & $\begin{array}{l}\text { Consumo entendido como un acto de intervención en el entorno, con consecuencias } \\
\text { sociales y ambientales. Organización de los propios criterios de compra. }\end{array}$ \\
\hline SE 5 & $x$ & $\begin{array}{l}\text { Consumo entendido como un acto de intervención en el entorno, con consecuencias } \\
\text { sociales y ambientales. Organización de los propios criterios de compra.(CE) }\end{array}$ \\
\hline CD 1 & $x$ & Organización de los propios criterios de compra.(CE) \\
\hline \multicolumn{3}{|l|}{$\mathrm{CD} 2$} \\
\hline \multicolumn{3}{|l|}{$\mathrm{CD} 3$} \\
\hline CD 4 & $x$ & $\begin{array}{l}\text { Consumo entendido como un acto de intervención en el entorno, con consecuencias } \\
\text { sociales y ambientales. Influencias externas a la hora de consumir.(CE) }\end{array}$ \\
\hline CD 5 & $x$ & Diversidad de estilos a la hora de vestir.(CE) \\
\hline
\end{tabular}

Figura 7. Tabla de análisis de datos de la guía docente de un taller de secundaria global para el ODS 12. 
En total, se han realizado 374 tablas de datos (22 talleres y 17 ODS). A continuación, se muestra un ejemplo de una de las tablas, donde se analiza la presencia del ODS 12 en el taller ¿Qué me pongo esta mañana? En ésta, se recogen los contenidos extraídos de la guía docente que cumplen los criterios anteriormente citados para ser aceptados como válidos en la relación con los Objetivos de Aprendizaje (OA) de este ODS (Figura 7).

Para completar el análisis, se han analizado las transcripciones de las entrevistas realizadas a los dos equipos de expertos junto con su diario de conclusiones para determinar que ODS se encuentran presentes en cada taller según su visión.

La presencia de los ODS se ha determinado a través de la unión de los datos procedentes de las tres fuentes. La triangulación de los datos permite validar con rigor los resultados, así como comprender las causas de inclusión o exclusión de cada ODS en los talleres. Esta presencia se ha valorado como presencia suficiente (validación por una fuente: guía, educadores o responsables), presencia notable ( validación por dos fuentes), presencia sobesaliente ( validación por todas las fuentes)

A continuación, se muestra un ejemplo de las tablas (Figuras 8) donde aparecen las coincidencias entre las tres fuentes en la presencia de un ODS concreto para el grupo de talleres globales de secundaria.

\begin{tabular}{|c|c|c|c|}
\hline \multirow{2}{*}{} & \multicolumn{3}{|c|}{ ODS 12 } \\
\cline { 2 - 4 } & Guía & Educadores & Responsables \\
\hline SG1 & $\mathrm{X}$ & & $\mathrm{X}$ \\
\hline SG2 & $\mathrm{X}$ & & $\mathrm{X}$ \\
\hline SG3 & $\mathrm{X}$ & & $\mathrm{X}$ \\
\hline SG4 & $\mathrm{X}$ & & $\mathrm{X}$ \\
\hline SG5 & $\mathrm{X}$ & & $\mathrm{X}$ \\
\hline SG6 & $\mathrm{X}$ & $\mathrm{X}$ & $\mathrm{X}$ \\
\hline
\end{tabular}

\begin{tabular}{|l|l|l|l|l|}
\hline \multicolumn{5}{|c|}{ ODS 12 } \\
\hline & ausencia & $\begin{array}{c}\text { Presencia } \\
\text { suficiente }\end{array}$ & $\begin{array}{c}\text { Presencia } \\
\text { notable }\end{array}$ & $\begin{array}{c}\text { Presencia } \\
\text { sobresaliente }\end{array}$ \\
\hline SG1 & & & & \\
\hline SG2 & & & & \\
\hline SG3 & & & & \\
\hline SG4 & & & & \\
\hline SG5 & & & & \\
\hline SG6 & & & & \\
\hline
\end{tabular}

Figura 8. Tratamiento de resultados de los talleres de secundaria globales para el ODS 12.

El mismo procedimiento se ha seguido para analizar las competencias transversales de aprendizaje para los ODS, las cuales vienen descritas en el documento de la UNESCO. En el caso de las entrevistas realizadas a los expertos, a través de la discusión han consensuado cuáles de las competencias se trabajan en cada uno de los talleres. En las guías docentes se han valorado los contenidos y las orientaciones didácticas descritas de cada taller para encontrar relaciones con las competencias siguiendo los criterios utilizados para valorar la presencia de los ODS.

\section{Resultados}

Los resultados se organizan en relación a los dos objetivos definidos: (1) describir la incorporación de los objetivos de aprendizaje en las actividades educativas de la Escuela del Consumo de Catalunya y (2) describir la incorporación de las competencias transversales en las actividades educativas. 
La descripción de la incorporación de los objetivos de aprendizaje en las actividades educativas de la Escuela del Consumo de Catalunya.

Los resultados mostrados en la figura 9 nos permiten realizar un análisis desde dos enfoques y cuestiones complementarias: ¿Qué ODS son mas incorporados? Y ¿Qué actividades educativas incorporan más ODS?

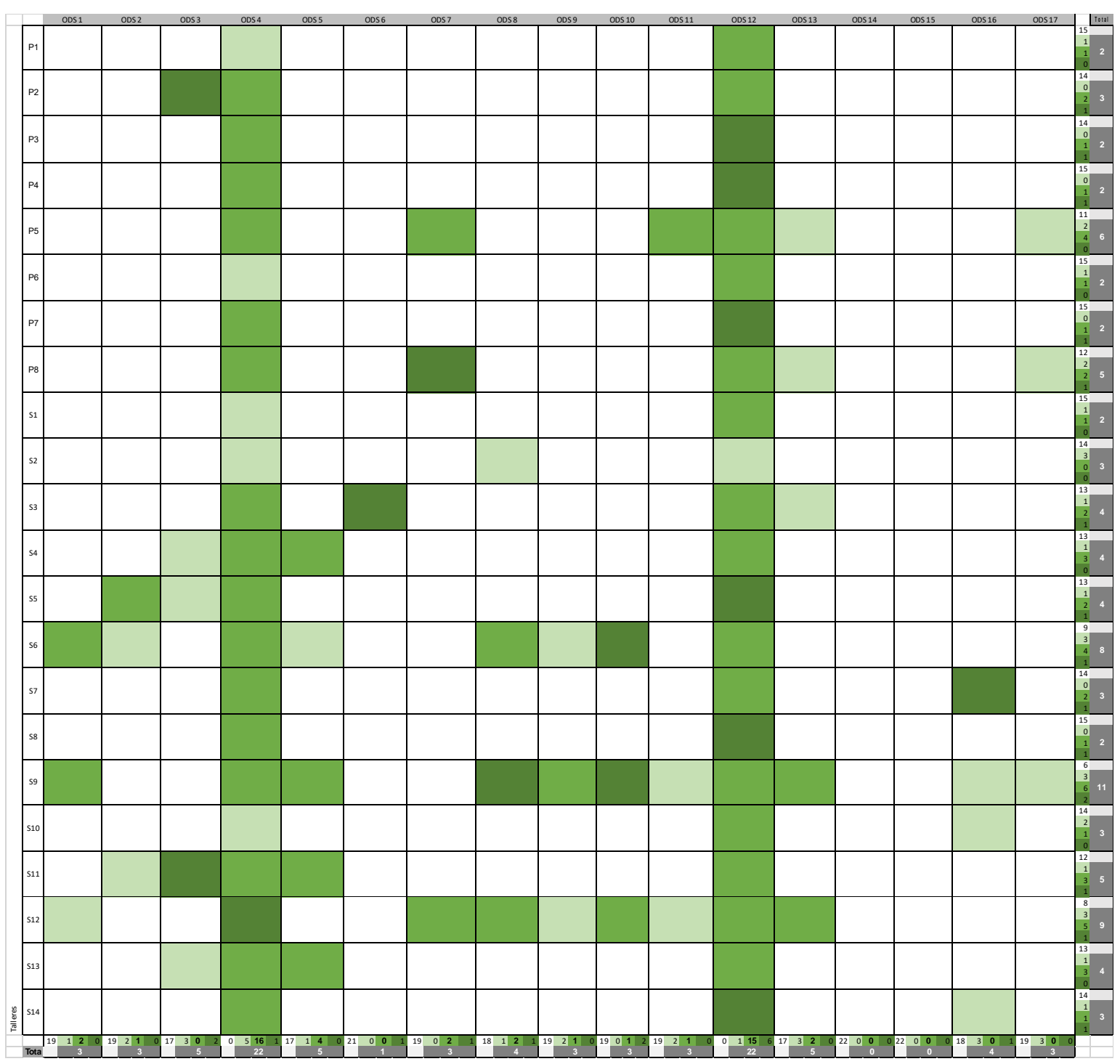

Figura 9. Tabla de representación de la presencia de los ODS en los talleres.

¿Qué ODS son mas incorporados?

Un primera aproximación global a los resultados nos indica que los diecisiete ODS se incorporan de forma diversa en la actividad educativa de la ECC pues hay dos ODS que no se incorporan, trece que se incorporan de forma diferente y dos que siempre se incorporan.

Los ODS en referencia a la biodiversidad terrestre y submarina (ODS 14 y 15) no són incorporados, un resultado que no sorprende pues si bien la educación del consumo conecta los productos y servicios producidos con el origen de los materiales y recursos naturales su enfoque no se orienta a combatir la pérdida de biodiversidad o la gestión de ecosistemas terrestres o marinos. 
El ODS 6 en relación a la gestión sostenible del agua únicamente se incorpora en un actividad educativa (¿El agua está siempre en equilibrio?) pero destaca su fuerte presencia por ser incorporada por los responsables del proyecto, el equipo educativo y las guías docentes.

Un resultado significativo es la incorporación de los ODS en relación a una educación de calidad (ODS 4) y un consumo y una producción responsable (ODS 12) en todas las actividades educativas que ofrece la ECC. En concreto el ODS 4 tiene una presencia suficiente de 4 , una presencia notable de 16 y una presencia sobresaliente de 1 . El ODS 12 tiene un a presencia suficiente de 1, una presencia notable de 15 y una presencia sobresaliente de 6.

En la forma diversa de incorporar el resto de ODS se observa que hay tres ODS que también se incorporan de forma adecuada por tener una presencia satisfactoria, notable o sobresaliente en cinco actividades educativas. En concreto en el ODS 5 de igualdad de género, el ODS 13 de acción climática y el ODS 3 de salud y bienestar.

Los resultados indican que las actividades educativas son coherentes con el proyecto educativo de la ECC y que la institución tiene una fuerte identidad en su cometido de educar en consumo. Un resultado que si bien parece obvio y redundante no lo es, pues los desajustes entre los objetivos institucionales, los programes educativos desarrollados y la inclusión de estos en el perfil de los educadores o formadores en ocasiones se convierte en una debilidad a la hora de impulsar proyectos de educación ambiental (Banqué, Calafell y Bonil, 2015). La fuerte incorporación de los ODS 4 y 12 indican que el programa de la ECC enseña a los diferentes participantes, jóvenes de secundaria y bachillerato y niños y niñas de primaria la importancia de los estilos de vida en relación al consumo y su papel como consumidores desde una metodología participativa y capaz de empoderarse para un futuro mas sostenible.

A la vez la forma de enfocar las actividades de educación del consumo conecta con un enfoque más humanístico y social como el hecho que la producción de productos y servicios tiene una fuerte componente para la oportunidad de género y la salud de las personas. Así como también una visión que conecta con cuestiones socialmente y científicamente emergentes y de actualidad como el efecto invernadero y la relación con los estilos de vida de consumo a nivel local y global.

¿Qué actividades educativas incorporan más ODS?

Globalmente los resultados indican que todos los talleres incorporan como mínimo dos ODS ( en la línea de los resultados anteriores el ODS 4 y el ODS 12) y que nueve de las veintidós actividades ofrecidas (41\%) no incorporan más ODS que éstos. El resto de actividades (59\%) incorporan en su diseño la relación entre tres ODS o más. En concreto destacan las actividades ¿Qué me pongo esta mañana? por incorporar once ODS y ¿El chocolate es dulce para todo el mundo? Por incorporar nueve ODS. Otras actividades educativas que destacan són: ¿ Cómo se mueve la energía por la escuela? Con seis ODS y las actividades ¿Qué valor tiene la energía que consumimos? $Y_{\text {¿Me }}$ siento bien?, con cinco ODS.

Los resultados muestran que casi todas las actividades que únicamente incorporan el ODS 4 y el ODS 12 corresponden a las propuestas ofrecidas a la etapa de educación primaria, excepto las dos actividades que toman como punto de partida la gestión de la energía en el consumo. Una evidencia que se puede explicar por la antigüedad de 
los talleres de educación primaria, que si bien se han reformulado, mantienen el diseño y las herramientas didácticas de cuando se ofrecieron por primera vez ( durante el curso 2006-2007) (Figura 10). En cambio las actividades sobre la gestión de la energía corresponden a actividades incorporadas en la oferta desde 2015, los cuales incorporan mejor la reflexión y avance práctico para desarrollar el modelo educativo de la idea vector y sus esferas y las estrategias metodológicas.

En referencia a las actividades con mayor presencia de distintos ODS se puede atribuir este resultado a que son actividades que trabajan fenómenos, preguntas y contenidos estrucurantes que abren a nuevas perspectivas y miradas y en las que necesariamente se ponen en juego diferentes disciplinas que aportan una visión interdisicplinaria que coincide con distintos ODS. Por ejemplo, la actividad ¿Qué me pongo esta mañana? trabaja a partir de un fenómeno como es la compra de ropa, contenidos estructurantes referidos al origen de la ropa que llega a la tienda y sus consecuencias de equidad social y de huella ecológica. La diversidad de estilos de moda y su presión en los jóvenes para pertenecer a un grupo identitario.

\begin{tabular}{|c|c|c|c|c|c|c|}
\hline \multirow{2}{*}{ Etapa } & \multirow[b]{2}{*}{ Taller } & \multirow{2}{*}{\begin{tabular}{|c|} 
Incorporación \\
de Modelo \\
idea vector y \\
sus esferas \\
\end{tabular}} & \multicolumn{4}{|c|}{ Herramientas metodológicas } \\
\hline & & & fenómeno & pregunta & $\begin{array}{c}\text { contenidos } \\
\text { estrucuturantes }\end{array}$ & $\begin{array}{c}\text { diálogo } \\
\text { disciplinario }\end{array}$ \\
\hline \multirow{8}{*}{$\begin{array}{c}\text { Etapa } \\
\text { primaria }\end{array}$} & Veo, veo... qué tienda es? & & $\mathrm{x}$ & $\mathrm{x}$ & $\mathrm{x}$ & $\mathrm{x}$ \\
\hline & Ñam, ñam...que hay para merendar? & & $\mathrm{x}$ & $\mathrm{x}$ & $\mathrm{x}$ & $\mathrm{x}$ \\
\hline & ¿Me hago mayor? & & $\mathrm{x}$ & $\mathrm{x}$ & $\mathrm{x}$ & $\mathrm{x}$ \\
\hline & ¿A que jugamos? & & $\mathrm{x}$ & $\mathrm{x}$ & $\mathrm{x}$ & $\mathrm{x}$ \\
\hline & ¿Como se mueve la energía por la escuela? & $\mathrm{x}$ & $\mathrm{x}$ & $\mathrm{x}$ & $\mathrm{x}$ & $\mathrm{x}$ \\
\hline & ¿Qué hacemos esta tarde? & & $\mathrm{x}$ & $\mathrm{x}$ & $\mathrm{x}$ & $\mathrm{x}$ \\
\hline & ¿Me compras una sonrisa? & & $\mathrm{x}$ & $\mathrm{x}$ & $\mathrm{x}$ & $\mathrm{x}$ \\
\hline & ¿Qué valor tiene la energía que consumimos? & $\mathrm{x}$ & $\mathrm{x}$ & $\mathrm{x}$ & $\mathrm{x}$ & \\
\hline \multirow{14}{*}{$\begin{array}{c}\text { Etapa } \\
\text { secundaria }\end{array}$} & ¿Me sirven los servicios? & & $\mathrm{x}$ & $\mathrm{x}$ & $\mathrm{x}$ & \\
\hline & ¿Dónde tengo mi dinero? & & $\mathrm{x}$ & $\mathrm{x}$ & $\mathrm{x}$ & $\mathrm{x}$ \\
\hline & ¿El agua está siempre en equilibrio? & $\mathrm{x}$ & $\mathrm{x}$ & $\mathrm{x}$ & $\mathrm{x}$ & $\mathrm{x}$ \\
\hline & ¿Mensajes o mensajeros? & & $\mathrm{x}$ & $\mathrm{x}$ & $\mathrm{x}$ & $\mathrm{x}$ \\
\hline & ¿Cómo sé que compro? & & $\mathrm{x}$ & $\mathrm{x}$ & $\mathrm{x}$ & \\
\hline & ¿El chocolate es dulce para todos? & $\mathrm{x}$ & $\mathrm{x}$ & $\mathrm{x}$ & $\mathrm{x}$ & $\mathrm{x}$ \\
\hline & ¿Qué reglas tiene el consumo? & & $\mathrm{x}$ & $\mathrm{x}$ & $\mathrm{x}$ & \\
\hline & ¿Vamos de compras? & & $\mathrm{x}$ & $\mathrm{x}$ & $\mathrm{x}$ & $\mathrm{x}$ \\
\hline & ¿Qué me pongo esta mañana? & $\mathrm{x}$ & $\mathrm{x}$ & $\mathrm{x}$ & $\mathrm{x}$ & $\mathrm{x}$ \\
\hline & Es sábado, ¿qué hacemos? & & $\mathrm{x}$ & $\mathrm{x}$ & $\mathrm{x}$ & $\mathrm{x}$ \\
\hline & ¿Me siento bien? & $\mathrm{x}$ & $\mathrm{x}$ & $\mathrm{x}$ & $\mathrm{x}$ & $\mathrm{x}$ \\
\hline & ¿Siempre vemos la energía que consumimos? & $\mathrm{x}$ & $\mathrm{x}$ & $\mathrm{x}$ & $\mathrm{x}$ & $\mathrm{x}$ \\
\hline & ¿Impactos o mensajes? & $\mathrm{x}$ & $\mathrm{x}$ & $\mathrm{x}$ & $\mathrm{x}$ & $\mathrm{x}$ \\
\hline & Internet: ¿consumimos o nos consumen? & $\mathrm{x}$ & $\mathrm{x}$ & $\mathrm{x}$ & $\mathrm{x}$ & $\mathrm{x}$ \\
\hline
\end{tabular}

Figura 10. Incorporación del modelo didáctico idea vector y las herramientas metodológicas ( fenómeno, preguntas, ejes estructurantes y diálogo disciplinario) en el diseño de los talleres

\section{La descripción de la incorporación de las competencias transversales en las actividades educativas de la Escuela del Consumo de Catalunya.}

Los resultados mostrados en la figura 11 nos permiten realizar un análisis desde dos enfoques y cuestiones complementarias: ¿Qué competencias transversales son mas 
incorporadas? Y ¿Qué actividades educativas incorporan más competencias transversales?

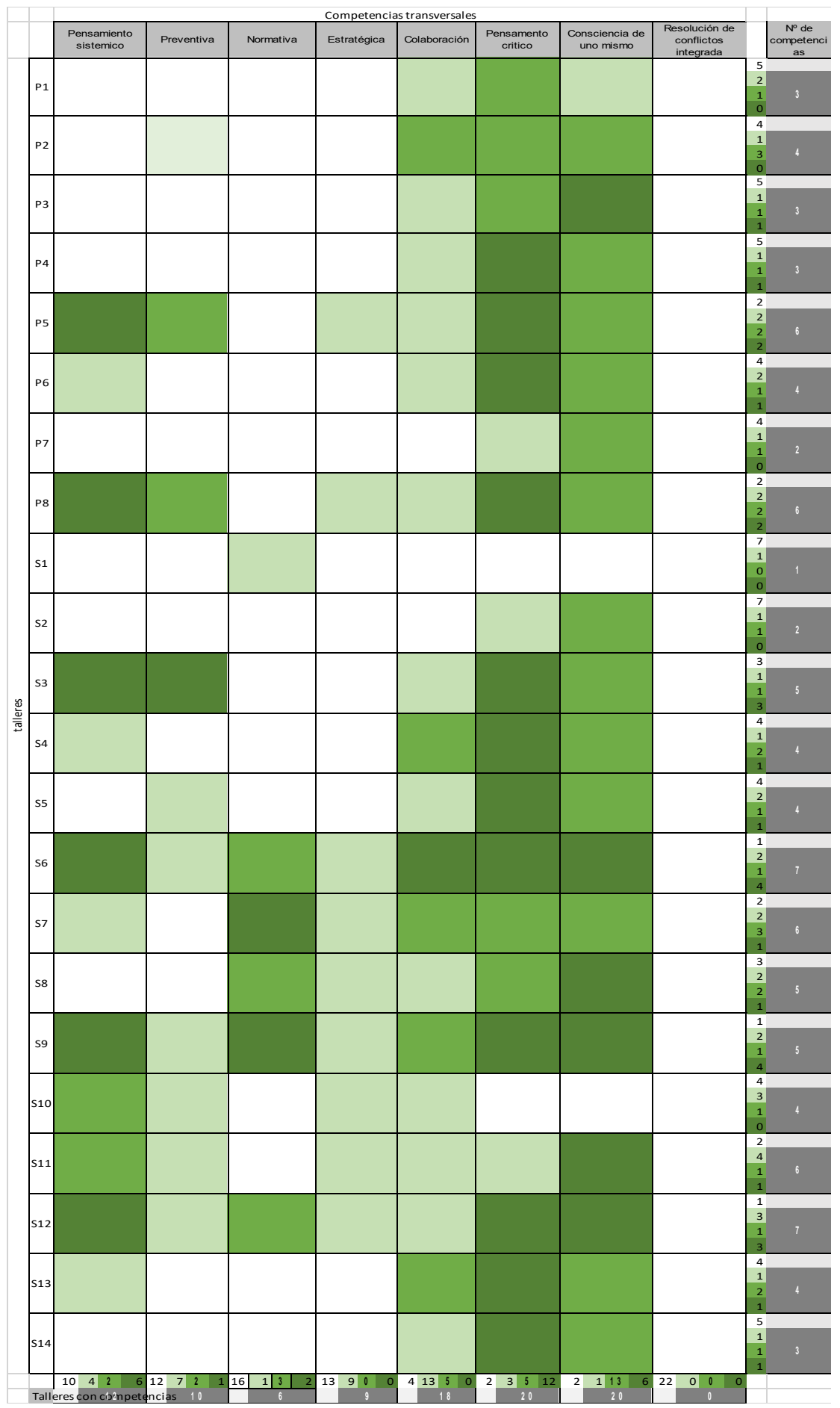

Figura 11. Tabla de representación del grado de presencia de las competencias transversales en los talleres.

¿Qué competencias transversales son mas incorporadas?

Los resultados indican que únicamente la competencia de resolución de conflictos no se incorpora en las actividades educativas y que el resto de las competencias son incorporadas aunque de forma diversa. 
Las dos competencias más incorporadas son pensamiento crítico y consciencia de uno mismo. Las competencias de pensamiento sistémico y colaboración también son significativas y es muy débil la incorporación de la competencia preventiva, normativa y estratégica.

Un primer resultado es que hay un patrón claro y que conecta claramente con el propósito de la escuela del consumo : educación responsable (tomar consciencia de los actos de consumo y la implicación del consumidor), crítica (actuar desde el análisis y la reflexión) y activa (tomar decisiones y acciones).

Así como también una coherencia entre el modelo didáctico propuesta que se focaliza en la complejidad y el diálogo entre diversidad de miradas, una evidencia que se observa en la incorporación del pensamiento sistémico y la competencia colaborativa.

Sorprendentemente no incorpora unas competencias más propias de la educación del consumo tradicional como es la normativa y la preventiva. En el caso de la competencia estratégica sí que se observa cierta disfuncionalidad con la propuesta pretendida en la que el consumidor se convierte en un ciudadano con una fuerte componente estratega ante la sociedad de consumo y por tanto, esta competencia se convierte en un elemento a poner atención.

¿Qué actividades educativas incorporan más competencias transversales?

Los talleres que incorporan más competencias son: ¿El chocolate es dulce para todo el mundo? Y ¿Siempre vemos la energía que consumimos?, ya que incorporan todas las competencias transversales excepto la competencia de resolución de conflictos integrada.

Otras actividades como, ¿Me siento bien? ¿Cómo se mueve la energía por la escuela? Y ¿Cuál es el valor de la energía que consumimos? Incorporan seis competencias transversales: pensamiento sistémico, prevención, estratégica, colaboración, pensamiento crítico, consciencia de uno mismo y ¿Qué reglas tiene el consumo? Incluye la competencia normativa en vez de la competencia preventiva. También son significativas por la incorporación de cinco competencias transverales las actividades ¿Vamos de compras?, ¿Qué me pongo esta mañana? y ¿El agua esta siempre en equilibro?

Estas actividades educativas en mayor parte corresponden a actividades que incorporan en su diseño tanto el modelo didáctico de la idea vector como las herramientas metodológicas asociadas (figura 9).

\section{Conclusiones}

La investigación en torno a la presencia de los ODS en el programa educativo de la ECC ofrece una diagnosis interesante en relación a las actividades de educación del consumo que se ofrecen y en referencia al diseño de sus actividades desde el modelo didáctico de la idea vector y sus esferas.

En relación a los ODS el 59\% de las actividades ofrecidas des de la ECC contemplan tres o más ODS en su diseño. Uno de los atributos de los ODS es la importancia de vincular de forma transversal cada uno de ellos y esta contribución, desde nuestro punto de vista es esencial para aproximarse a la educación ambiental desde una visón compleja e interdisciplinaria que se da en el programa educativo de la ECC. A la vez la 
forma como se incorporan los ODS en la ECC evidencian un patrón claro, identitario y bien acotado de lo que se entiende por consumo y por educación. Al mismo tiempo, los resultados favorecen la toma decisiones hacia una proyección futura de la actividad como la necesidad de rediseñar la mayoría los talleres que se ofrecen a la etapa de educación primária según el modelo de idea vector y sus esferas para que incorporen una visión más interdisciplinária y compleja de los ODS y las competencias transversales. También, la necesidad de reflexión la ideoneidad o no de trabajar competencies transversales que son poco incorporadas como La resolución de conflictos integrada, la preventiva, la normativa y la estratégica.

Las competencias en torno al pensamiento crítico, la consciencia de uno mismo y el pensamiento sistémico han sido tradiconalmente un hándicap para la Educación Ambiental. No tanto en su reconocimiento sinó en la definición de modelos didácticos y metodologías efectivas para diseñar e implementar acciones educativas con estos enfoques.

En este sentido el modelo didáctico de la idea vector y sus esferas se convierte en una propuesta pontente para trabajar estas competencias, pues la investigación muestra como son bien incorporadas en el diseño de actividades. Para fortalecer esta propuesta convendría profundizar en la investigación y ampliar una cuarta fuente: la visión del alumnado. De esta forma se podría indagar si dichas competencias son aprendidas por los participantes.

El caso que se ha mostrado pensamos que es un avance también en la conexión entre la investigación y la actividad educativa ya que la ECC ha favorecido promover espacios de reflexión sobre la práctica cotidiana. Se muestra así que la interacción entre instituciones, en este caso entre el gobierno de Catalunya y la investigación de la universidad favorece a indagar y avanzar en el cómo diseñar proyectos de educación ambiental desde la rigurosidad y la cotidianidad.

\section{Agradecimientos}

El artículo se ha desarrollado gracias al servicio educativo de l'Escola del Consum de Catalunya de l'Agència Catalana del Consum (Generalitat de Catalunya).

La Investigación que se presenta es resultado del Trabajo Final del Programa Máter Universitario de Investigación en Educació, especialidad Educación Científica y Matemática de la Universitat Autònoma de Barcelona "Una mirada a los Objetivos de Desarrollo Sostenible: descripción y caracterización de la incorporación de los ODS en la oferta educativa de la Escuela del Consumo de Cataluña.

\section{Referencias}

Arnal, J. (2000) Metodologies de la investigación educativa. In J.Mateo Andrés \& MC Vidal Xifre (Eds), Mètodes d'investigació en educació. Barcelona: Universitat Oberta de Catalunya.

Betrián, E., Galitó, N., García, N., Jové, G., Macarulla, M. (2013). La triangulación múltiple como estrategia metodológica. REICE. Revista Iberoamericana sobre Calidad, Eficacia y Cambio en Educación, 11(4), 5-24. ISSN: 1696-4713.

Banqué, N., Querol, M. y Calafell, G. (2011). Em compres un somriure? Una proposta per treballar l'educació del consum a l'aula. Perspectiva escolar, 352, 51-55. 
Banqué, N., Bonil, J. y Calafell, G. (2015). Dibuixant cartografies. Un perfil competencial que afavoreix la innovació d'equips educatius en contextos e ciència. Tesi doctoral, Universitat Autònoma de Barcelona.

Bonil, J., Calafell, G., Granados, J., Junyent, M., y Tarín, M. R. (2012). Un modelo formativo para avanzar en la ambientalización curricular. Profesorado. Revista de Currículum y Formación de Profesorado, 16(2), 145-163.

Bonil, J., Calafell, G., Fonolleda, M., Querol, M., Pujol, R.M. (2013). À la recherche de pistes pour réaliser l'éducation a' la consommation dans un monde en changement. Dans Agundez, A. y Jutras, F. (dir.), Enseigner et éduquer à la consommation. 185-202.

Calafell, G. (2007). El diálogo disciplinar como herrramienta para diseñar islotes de racionalidad. Encuentros multidisciplinares, 9 (25), 58-65. ISSN-e1139-9325.

Calafell, G. y Junyent, M. (2017). La idea vector y sus esferas: una propuesta formativa para la ambientalización curricular desde la complejidad. Teoría de la educación, 29, 189-216.

Calafell, G., Junyent, M., Bonil, J. (2015). Una propuesta para ambientalizar el currículum. Cuadernos de Pedagogía, 460, 56-60.

Calafell, G., Banqué, N. y Viciana, S. (2019). Com rutlla l'energia que consumim a l'escola?. Perspectiva escolar, (en edición final).

Calafell, G. y Bonil, J. (2010). Em sento bé? Bifstek, pírcings i altre decisons. Educació i sostenibilitat, 9, 8-11.

Colás Bravo, MP y Buendía Eisman, L. (1998) Investigación educativa. Sevilla: Alfar.

CNEA (2011). Ponencias del Congreso Nacional de Educación Ambiental. https://www.cnea.cat/copia-de-ponents (consulta 17 de febrero del 2019).

Develay, M. (1995). De l'apprentissage à l'enseignement. Pour une épistémologie scolaire. Paris: ESF éditeur.

Gómez, C. (2017/2018). Objetivos de desarrollo sostenible (ODS): una revisión crítica. Papeles de relaciones ecosociales y cambio global, 140, 107-118.

Gómez, R.; Fonolleda,M. y Calafell (2013). A què juguem? Un taller per treballar el joc i les joguines. Perspectiva escolar,367, 65-69.

Gutierrez Bastida, J.M. (2018). Education ambientalis. Invitación a la educación ecosocial en el Antropoceno. Madrid: Editorial Bubok.

Grau, Q. (2018). Una mirada als Objectius de Desenvolupament Sostenible: descripció i caracterització de la incporporació dels ODS a l'oferta educativa de l'Escola del Consum de Catalunya. Trabajo de Fin de Master.

Márquez, C., Roca, M., Gómez, A., Sardá, A., Pujol, R.M. (2004). La construcción de modelos explicativos complejos mediante preguntas mediadoras. Investigación en la escuela, 53, 71-81.

Organización de las Naciones Unidas para la Educación, la Ciéncia y la Cultura (2017). Educación para los objectivos de desarrollo sostenible: objetivos de aprendizaje. París: UNESCO. 
Rodríguez, F., García J.E. (2011). ¿Qué diferencias hay entre el conocimiento cotidiano y el conocimiento científico de docentes en formación sobre el concepto de energía?. Investigación en la escuela, 75, 63-71.

Rodríguez, G. y Gutiérrez, J. (2005) Un modelo de estudios empíricos en investigación educativa mediante procedimientos de triangulación. Revista Portuguesa de Pedagogía, 39 (1)(0870-0418), 135-157.

Sauvé, L., Villemagne, C. (2015). La ética ambiental como proyecto de vida y "obra" social: Un desafío de formación CPU-e, Revista de Investigación Educativa, 21, 188-209.

Tilbury, D (20011). Reconceptualizando la educación ambiental para un nuevo siglo. Tópicos en educación ambiental, 3(7), 65-73.

Wals, A.E.J., Stevenson, R., Brody, M. i Dillon, J. (2013). Tentative Directions for Environmental Research in Uncertain Times. International Handdbook of Research on Environmental Education. American Education Research Associaton. pp.542-549. 